\title{
Three channel model of meson-meson scattering and scalar meson spectroscopy
}

\author{
R. Kamiński ${ }^{1}$, L. Leśniak ${ }^{2}$ \\ Department of Theoretical Physics \\ H. Niewodniczański Institute of Nuclear Physics, \\ PL 31-342 Kraków, Poland \\ and \\ B. Loiseau \\ Division de Physique Théorique*, Institut de Physique Nucléaire, \\ F-91406, Orsay Cedex \\ and \\ LPTPE Université P. \& M. Curie, 4 Place Jussieu, \\ F-75252, Paris Cedex 05, France
}

July 13, 2021

\begin{abstract}
New solutions on the scalar - isoscalar $\pi \pi$ phase shifts are analysed together with previous $K \bar{K}$ results using a separable potential model of three coupled channels $(\pi \pi, K \bar{K}$ and an effective $2 \pi 2 \pi$ system). Model parameters are fitted to two sets of solutions obtained in a recent analysis of the CERN-Cracow-Munich measurements of the $\pi^{-} p_{\uparrow} \rightarrow \pi^{+} \pi^{-} n$ reaction on a polarized target. A relatively narrow $(90-180 \mathrm{MeV})$ scalar resonance $f_{0}(1400-1460)$ is found, in contrast to a much broader $(\Gamma \approx 500 \mathrm{MeV})$ state emerging from the analysis of previous unpolarized target data.
\end{abstract}

The spectrum of scalar mesons is still not well known [1], 2]. The $J^{P C}=0^{++}$ meson nonet is not free from ambiguities and the internal structure of scalar mesons is rather controversial. Recently, many papers have been devoted to the study of the lowest scalar glueball [3-6]. In these articles properties of the $f_{0}(1500)$ resonance, observed recently by the Crystal Barrel Collaboration in $p \bar{p}$ annihilations [7, 8], are analysed and interpreted. Furthermore, lattice QCD calculations predict the mass of the lowest scalar glueball to fall between 1500 and $1700 \mathrm{MeV}$ [9, 10]. One should also mention increasing evidence for a broad scalarisoscalar resonance $\sigma$ [11-14] below $1000 \mathrm{MeV}$. This state reappeared as $f_{0}(400-$ 1200) in the last edition of the Particle Data Group [1], after 22 years of absence. 
Well established resonance $f_{0}(980)$ lies at the $K \bar{K}$ threshold. Its interpretation, however, is a subject of lively discusions, as can be seen for example in Ref. [15] and articles cited therein.

In Ref. [11] the scalar meson spectrum was studied in terms of a relativistic $\pi \pi$ and $K \bar{K}$ coupled channel model from the $\pi \pi$ threshold up to $1400 \mathrm{MeV}$. The phenomenological parameters were constrained by fitting the $S$-wave data extracted from the experimental cross sections on the $\pi^{+} \pi^{-}$production by $\pi^{-}$ scattering on unpolarized hydrogen target [16]. Further constraints were imposed by the $K \bar{K}$ phase shift analysis of Ref. [17.

Recently, authors of Ref. [18 have analysed data obtained on a polarized target by the CERN-Cracow-Munich group for the $\pi^{-} p_{\uparrow} \rightarrow \pi^{+} \pi^{-} n$ reaction [19]. Separation of the $\pi$ and $a_{1}$ exchange amplitudes in this reaction was then possible for the first time, using assumptions much weaker than in all previous analyses. From a set of four solutions for the isoscalar $S$-wave phase shifts up to 1600 $\mathrm{MeV}$, two of them ("down-flat" and "up-flat") satisfy the unitarity constraint. The "down-flat" solution is in good agreement with the former solution of Ref. [16] up to $1400 \mathrm{MeV}$. Above $1400 \mathrm{MeV}$ one observes an increase of the phase shifts and larger inelasticies than those in Ref. [16]. This could be a manifestation of the presence of scalar mesons $f_{0}(1370)$ or $f_{0}(1500)$ in that energy range. There, a strong four-pion production has been observed in different experiments $[7,8,20-$ 22 . This provides a compelling argument to take into account the $4 \pi$ channel. In this channel there is some evidence of clustering into $\sigma \sigma$ or $\rho \rho$ pairs [6-9]. In [23] an attempt was made to describe the $\pi \pi S$-wave in the 1000 to $2000 \mathrm{MeV}$ region by generalization of the non-relativistic 2-channel model 24] to three coupled channels.

In the present paper we extend the isospin $0 S$-wave relativistic $2-$ channel model of Ref. [11] by adding to its $\pi \pi$ and $K \bar{K}$ channels an effective third coupled channel, here called $\sigma \sigma$.

We consider the $S$-wave scattering and transition reactions between three coupled channels of meson pairs labelled 1, 2 and 3. Reaction amplitudes $T$ satisfy a system of coupled channel Lippmann-Schwinger equations [11]:

$$
<\mathbf{p}|T| \mathbf{q}>=<\mathbf{p}|V| \mathbf{q}>+\int \frac{d \mathbf{u}}{(2 \pi)^{3}}<\mathbf{p}|V| \mathbf{u}><\mathbf{u}|G| \mathbf{u}><\mathbf{u}|T| \mathbf{q}>,
$$

where $V, G$ and $T$ are $3 \times 3$ matrices, $V$ is the interaction matrix and $G$ is the diagonal matrix of channel propagators:

$$
G_{j}(E, \mathbf{u})=\frac{1}{E-2 E_{j}(\mathbf{u})+i \epsilon}, \quad \epsilon \rightarrow 0(+), \quad j=1,2,3 .
$$

In Eq. (2) $E$ is the total energy, $E_{j}=\sqrt{m_{j}^{2}+\mathbf{u}^{2}}$ and $m_{j}$ is the meson mass in channel $j$. We consider meson pairs of same mass and momentum $\mathbf{u}$ in their centre of mass system. 
We choose a separable form of the interaction:

$$
<\mathbf{p}\left|V_{\alpha \gamma, j}\right| \mathbf{q}>=\sum_{j=1}^{n} \lambda_{\alpha \gamma, j} g_{\alpha, j}(\mathbf{p}) g_{\gamma, j}(\mathbf{q}), \quad \alpha, \gamma=1,2,3
$$

where $\lambda_{\alpha \gamma, j}$ are coupling constants and

$$
g_{\alpha, j}(\mathbf{p})=\sqrt{\frac{4 \pi}{m_{j}}} \frac{1}{\mathbf{p}^{2}+\left(\beta_{\alpha, j}\right)^{2}}
$$

are form factors which depend on the relative centre of mass meson momenta $\mathbf{p}$ in the final channel or $\mathbf{q}$ in the initial channel. In the $\pi \pi$ channel $(j=1)$ we choose a rank-2 separable potential $(n=2)$ and in the other channels, i.e. $K \bar{K}(j=2)$ and $\sigma \sigma(j=3)$, a rank-1 potential $(n=1)$.

Following the formalism developed in Ref. [11] we can solve the system of equations (1) - (4), which leads to the Jost function

$$
D\left(\mathbf{k}_{1}, \mathbf{k}_{2}, \mathbf{k}_{3}\right)=\operatorname{det}(1-\lambda I)
$$

where $\lambda$ and $I$ are symmetric $4 \times 4$ matrices

$$
\lambda=\left(\begin{array}{cccc}
\lambda_{11,1} & 0 & \lambda_{12,1} & \lambda_{13,1} \\
0 & \lambda_{11,2} & \lambda_{12,2} & \lambda_{13,2} \\
\lambda_{12,1} & \lambda_{12,2} & \lambda_{22,1} & \lambda_{23,1} \\
\lambda_{13,1} & \lambda_{13,2} & \lambda_{23,1} & \lambda_{33,1}
\end{array}\right), I=\left(\begin{array}{cccc}
I_{1,11} & I_{1,12} & 0 & 0 \\
I_{1,12} & I_{1,22} & 0 & 0 \\
0 & 0 & I_{2,11} & 0 \\
0 & 0 & 0 & I_{3,11}
\end{array}\right)
$$

and

$$
I_{\alpha, i j}\left(\mathbf{k}_{\alpha}\right)=\int \frac{d \mathbf{u}}{(2 \pi)^{3}} g_{\alpha, i}(\mathbf{u}) G_{\alpha}(E, \mathbf{u}) g_{\alpha, j}(\mathbf{u}) .
$$

In (5) and (7) $\mathbf{k}_{\alpha}$ are the on-shell momenta connected with the total energy by

$$
E=2 \sqrt{\mathbf{k}_{1}^{2}+m_{1}^{2}}=2 \sqrt{\mathbf{k}_{2}^{2}+m_{2}^{2}}=2 \sqrt{\mathbf{k}_{3}^{2}+m_{3}^{2}}
$$

Analytical expressions for $I_{\alpha, i j}\left(\mathbf{k}_{\alpha}\right)$ integrals are given in Appendix A of Ref. [11]. Altogether, this model has 13 parameters: 9 coupling constants $\lambda_{\alpha \gamma, j}$ and 4 range parameters $\beta_{\alpha, j}$ (for $\alpha=1 j=1$ or 2 and for $\alpha \neq 1 j=1$ ). In the calculations we shall use dimensionless coupling constants defined as

$$
\Lambda_{\alpha \gamma, j}=\frac{\lambda_{\alpha \gamma, j}}{2\left(\beta_{\alpha, j} \beta_{\gamma, j}\right)^{3 / 2}}
$$

From now on, we will omit index $j$ for $\lambda_{\alpha \gamma, j}$ if both $\alpha$ and $\gamma$ are different from 1 and for $\beta_{\alpha, j}$ if $\alpha \neq 1$. 
$S$-matrix elements $S_{\alpha \beta}(\alpha, \beta=1,2,3)$ can be written in terms of the Jost function of different arguments, for example

$$
S_{11}=\frac{D\left(-\mathbf{k}_{1}, \mathbf{k}_{2}, \mathbf{k}_{3}\right)}{D\left(\mathbf{k}_{1}, \mathbf{k}_{2}, \mathbf{k}_{3}\right)}
$$

Further expressions for some other matrix elements can be found in Ref. [25]. The model satisfies the unitarity condition $S^{+} S=1$. Diagonal matrix elements are parametrized as

$$
S_{j j}=\eta_{j} e^{2 i \delta_{j}}, \quad j=1,2,3,
$$

where $\eta_{j}$ and $\delta_{j}$ are channel $j$ inelasticities and phase shifts, respectively. Expressions for nondiagonal elements can be found in [25]. Some of the $S$-matrix poles in the complex energy plane can be interpreted as resonances. They correspond to the zeroes of the Jost function $D\left(\mathbf{k}_{1}, \mathbf{k}_{2}, \mathbf{k}_{3}\right)$ as can be seen from (10).

We fit the existing experimental results on the $\pi \pi S$-wave isoscalar phase shifts together with inelasticity in the $\pi \pi$ channel and with the $K \bar{K}$ phase shifts. In this analysis we extend the 2-channel model developed in Ref. 111 since new data have been recently obtained [18] in a wider mass range (up to $1600 \mathrm{MeV}$ ). We choose a method based on the $\chi^{2}$ fit:

$$
\begin{gathered}
\chi^{2}=\chi_{\pi}^{2}+\chi_{\pi K}^{2}+\chi_{\eta}^{2}, \\
\chi_{\pi}^{2}=\sum_{l=1}^{N_{\pi}}\left|\frac{e^{2 i \delta_{\pi \pi}^{t h}}-e^{2 i \delta_{\pi \pi}^{e x p}}}{2 \Delta \delta_{\pi}}\right|^{2}, \\
\chi_{\pi K}^{2}=\sum_{l=1}^{N_{\pi K}}\left|\frac{e^{2 i\left(\delta_{\pi \pi}^{t h}+\delta_{K K}^{t h}\right)}-e^{2 i\left(\delta_{\pi \pi}^{e x p}+\delta_{K K}^{e x p}\right)}}{2 \Delta\left(\delta_{\pi \pi}+\delta_{K K}\right)}\right|^{2}, \\
\chi_{\eta}^{2}=\sum_{l=1}^{N_{\eta}}\left(\frac{\eta_{\pi \pi}^{t h}-\eta_{\pi \pi}^{e x p}}{\Delta \eta_{\pi \pi}}\right)^{2},
\end{gathered}
$$

where $\delta_{\pi \pi}, \delta_{K K}$ are phase shifts in channels 1 and $2, \Delta \sigma_{\pi}, \Delta \sigma_{K}$ are the corresponding experimental errors and $\eta_{\pi \pi}, \Delta \eta_{\pi \pi}$ are inelasticities and inelasticity errors, respectively. Superscripts "th" or "exp" refer to theoretical or experimental values. In Eqs. (13) to (15) $N_{\pi}, N_{\pi K}$ and $N_{\eta}$ are the numbers of experimental points.

Below $600 \mathrm{MeV}$ we use data from the $K_{e 4}$ decay [26] and from Refs. [27] and [28]. Above $600 \mathrm{MeV}$ we use the "down-flat" and "up-flat" solutions of the analysis of Ref. [18] on a polarized target. Constraints in the $K^{+} K^{-}$channel are also needed. Therefore, we have used the results of the analysis of reactions $\pi^{-} p \rightarrow K^{+} K^{-} n$ and $\pi^{+} n \rightarrow K^{-} K^{+} p$ [17], although targets were unpolarized there.

We begin with a simpler $\chi^{2}$-minimization by considering only the $\pi \pi$ and $K \bar{K}$ 2 -channel case. Starting parameters of the interaction potentials have been taken from 2 -channel fits obtained in [29]. In this case there are 8 free parameters, as 
indicated in Table 1. The corresponding fits for $\delta_{\pi \pi}, \eta_{\pi \pi}$ and $\varphi_{\pi K}=\delta_{\pi \pi}+\delta_{K K}$, plotted as dotted lines, are compared to the experiment in Figs. 1, 2 and 3. Above $1400 \mathrm{MeV}$ both "down-flat" and "up-flat" data indicate a decrease towards small values of $\eta(\eta \approx 0.6$ to 0.7$)$, albeit with large errors. Furthermore, it can be seen in Fig. 2 that the errors of $\eta$ are much larger than dispersion of their values. Therefore, in order to reproduce more easily this trend of $\eta$ as a function of energy, for most of the data points we reduce in Eq. (15) the experimental $\eta$ errors to 0.1. For the "down-flat" solution we keep, however, the original errors at 1310, 1470 and $1570 \mathrm{MeV}$ since inelasticities at these energies are above or close to 1 . For the last point at $1590 \mathrm{MeV}$ we increase the error from 0.41 to 1 since $\eta$ is as large as 1.52. Similarly, for the "up-flat" solution we do not change the errors in the range between 1350 and $1450 \mathrm{MeV}$, however, at 1310, 1470 and $1590 \mathrm{MeV}$ we increase the errors to 0.8 since $\eta$ values at those energies are substantially higher than 1.

We found that in the 2-channel case for the "down-flat" solution it was not possible to obtain the $\eta$ values with such modified errors between 1400 to 1600 $\mathrm{MeV}$ (this is slightly different for the "up-flat" solution as can be seen in Fig. $2 \mathrm{~b})$. In the 3-channel model, however, we can get a substantial decrease of $\eta$ above $1400 \mathrm{MeV}$ (see Fig. 2a). In order to achieve this behaviour, couplings between the $\pi \pi$ and $\sigma \sigma$ or $K \bar{K}$ channels should be sizable. The corresponding 14 free parameters including the $m_{3}$ mass for two of our best fits are given in Table 1, and their $\chi^{2}$ in Table 2. The 3-channel fits for the "down-flat" data are labelled by A and B, and those corresponding to the "up-flat" data - by C and $\mathrm{D}$, respectively. The fits favour $m_{3}$ masses in the range of 675 to $733 \mathrm{MeV}$. The values of $\delta_{\pi \pi}, \eta_{\pi \pi}$ and $\varphi_{\pi K}$ are drawn as thick and thin solid lines in Figs. 1-3 and compared to data. In Fig. 1 we have shown fits $\mathrm{A}$ and $\mathrm{C}$ only since energy dependence of fit $\mathrm{B}$ is very close to that of $\mathrm{A}$, and that of $\mathrm{D}$ - very close to $\mathrm{C}$. In Fig. 1 one can already notice a better agreement with data of the 3-channel model in comparison with the 2 -channel one. This is especially well visible in Fig. 1b. The main difference between the 2- and 3-channel fits lies in $\eta$ above 1400 $\mathrm{MeV}$, where the opening of the $\sigma \sigma$ channel leads to a fast decrease of inelasticity parameters (see Fig. 2a). Let us also note an improvement in $\varphi_{\pi K}$ over the 1000 to $1200 \mathrm{MeV}$ range, as can be seen in Fig. 3 and in Table 2 with a better $\chi_{\pi K}^{2}$.

Fits of similarly good quality were obtained with very different physical parameters in the $K \bar{K}$ and $\sigma \sigma$ channels. For example, in the $2-$ channel fits and in fit $\mathrm{A}$, the $K \bar{K}$ interaction is attractive while in the other cases it is repulsive (Table 1). Similarly, interchannel couplings are very different in both cases. In fit $\mathrm{A}$ we see rather strong, and in fit $\mathrm{C}$ very strong $\pi \pi$ to $\sigma \sigma$ and $K \bar{K}$ to $\sigma \sigma$ couplings, while in the $\mathrm{B}$ and $\mathrm{D}$ cases the $\pi \pi-K \bar{K}$ coupling, $\Lambda_{12,2}$, is particularly strong. Lack of a sufficient number of observables and/or experimental precision, in particular in the effective $4 \pi$ channel, leads to the existence of several good sets of model parameters with quite different channel and interchannel interactions. Other 3-channel fits of reasonable quality with less than 14 parameters can be also obtained. 
We have studied positions of the $S$-matrix poles in the complex energy plane $(E=M-i \Gamma / 2)$. For the 3 -channel model there are 8 different sheets which correspond to different signs of imaginary parts of channel momenta $\left(I m p_{1}, I m p_{2}, I m p_{3}\right)$. For example, on the sheet denoted by (-- ) all imaginary parts are negative. In the case of the 2-channel model there are only 4 sheets labelled by signs of $I m p_{1}$ and $I m p_{2}$. Resonance parameters predicted by the 2- and 3-channel models are summarized in Table 3. At low energy we find a very broad $f_{0}(500)$ resonance (also called $\sigma$ meson). Since the "up-flat" data indicate a faster increase of the $\pi \pi$ phase shifts with energy than the "down-flat" data, the $\sigma$ meson appears in the first case at a mass higher by about $40 \mathrm{MeV}$ and with a width smaller by about $50 \mathrm{MeV}$. In the three channel fits the $f_{0}(980)$ resonance is seen in the vicinity of the $K \bar{K}$ threshold with a width of about 60 to $70 \mathrm{MeV}$. The relatively narrow state $f_{0}(1400)$ appears in the 3 -channel fits (see Table 3). Its mass varies from about $1400 \mathrm{MeV}$ to $1460 \mathrm{MeV}$. For the "down-flat" fits this resonance is narrower $(\Gamma \approx 100 \mathrm{MeV})$ on sheet $(--+)$ than on sheet $(---)$. This width is very close to values found by the Crystal Barrel Group (Refs. [7, 8]) but the resonance masses are smaller than their values close to about $1500 \mathrm{MeV}$. We should point out that a narrow $(\Gamma=65 \pm 10 \mathrm{MeV})$ scalar resonance at $M=1445 \pm 5$ $\mathrm{MeV}$ has been found by the WA91 group at CERN in central production of $4 \pi$ in high-energy $p p$ collisions [22].

Finally, let us mention an important difference between the fits presented here for data taken on a polarized target in comparison with those performed with data [16] obtained on a nonpolarized target. If we fit the data of [16] using our 3-channel model, we obtain a very wide resonance at $M=1521 \mathrm{MeV}$ of width $503 \mathrm{MeV}$. This means that the recent analysis of data 18] supplies some new information on the $f_{0}(1400)$ meson.

In conclusion, we have analysed the isoscalar S-wave $\pi \pi$ and $K \bar{K}$ scattering using the CERN-Cracow-Munich data [18] together with the data of [17,26-28] in the framework of the 2- and the 3-channel models of meson-meson scattering. All fits of the phase shifts analysis [18] indicate presence of a relatively narrow $(90-180 \mathrm{MeV})$ scalar resonance of mass $1400-1460 \mathrm{MeV}$. This resonance is quite compatible with recent observations of a possible scalar glueball at 1500 $\mathrm{MeV}$ with a width of $100 \mathrm{MeV}$.

We are indebted to D. V. Bugg for useful correspondence and to J. Kisiel, V. E. Markushin and K. Rybicki for fruitful discussions.

This work has been performed in the framework of the IN2P3 - Polish Laboratories Convention (project No 93-71) and partially supported by the Polish State Committee for Research (grants No 2 P03B 231 and No 2 P03B 020 12).

${ }^{1}$ E-mail: kaminski@solaris.ifj.edu.pl

${ }^{2}$ E-mail: lesniak@bron.ifj.edu.pl

* Unité de Recherche des Universités Paris 11 et Paris 6 Associée au CNRS 


\section{References}

[1] Particle Data Group, Phys. Rev. D54 (1996) 1.

[2] R. Landua, in Proc. of 28th Int. Conf. on High Energy Physics, Warsaw,Poland, July 25-31, 1996, eds. Z. Ajduk and A. K. Wróblewski (World Scientific, Singapore), vol. I, p. 3.

[3] C. Amsler et al., (Crystal Barrel group), Phys. Lett. B291 (1992) 347.

[4] C. Amsler and F. E. Close, Phys. Rev. D53 (1996) 295.

[5] A.V. Anisovich, V. V. Anisovich, and A. V. Sarantsev, Phys. Lett. B395 (1997) 123.

[6] D. V. Bugg et al., Phys. Lett. B353 (1995) 378.

[7] A. Abele et al., Phys. Lett. B385 (1996) 425.

[8] A. Abele et al., Phys. Lett. B380 (1996) 453.

[9] G. S. Bali et al., Nucl. Phys. Proc. Suppl. 53 (1997) 239.

[10] D. Weingarten, Nucl. Phys. B (Proc. Suppl.) 53 (1997) 232.

[11] R. Kamiński, L. Leśniak, and J.-P. Maillet, Phys. Rev. D50 (1994) 3145.

[12] S. Ishida et al., Prog. Theor. Phys. 95 (1996) 745.

[13] M. Harada, F. Sannino and J. Schechter, Phys. Rev. D54 (1996) 1991.

[14] N. A. Törnqvist and M. Roos, Phys. Rev. Lett. 76 (1996) 1575.

[15] M. P. Locher, V. E. Markushin and H. Q. Zheng, Paul Scherrer Institute preprint PSI-PR-97-13, hep-ph/9705230.

[16] G. Grayer et al., Nucl. Phys. B75 (1974) 189.

[17] D. Cohen et al., Phys. Rev. D22 (1980) 2595.

[18] R. Kamiński, L. Leśniak, and K. Rybicki, Z. Phys C74 (1997) 79.

[19] H. Becker et al., Nucl. Phys. B151 (1979) 46.

[20] M. Alston-Garnjost et al., Phys. Lett. B36 (1971) 152.

[21] R.M. Baltrusaitis et al., Phys. Rev. D33 (1986) 1222.

[22] F. Antinori et al., Phys. Lett. B353 (1995) 589.

[23] W.M. Kloet and B. Loiseau, Z. Phys. A353 (1995) 227. 
[24] F. Cannata, J.-P. Dedonder, and L. Leśniak, Phys. Lett. B207 (1988) 115.

[25] L. Leśniak, Acta Phys. Pol. B27 (1996) 1835.

[26] L. Rosselet et al., Phys. Rev. D15 (1977) 574.

[27] A.A. Belkov et al., Pisma Zh. Eksp. Teor. Fiz. 29 (1979) 652.

[28] V. Srinivasan et al., Phys. Rev. D12 (1975) 681.

[29] R. Kamiński, PhD thesis, Cracow 1996, unpublished. 
Figure 1: Energy dependence of $\pi \pi$ phase shifts: a) fit to "down-flat" data of [18], thick solid line corresponds to fit A of the 3-channel model and dotted line to the 2-channel model fit; b) fit to "up-flat" data of [18], thick solid line corresponds to fit $\mathrm{C}$ of the 3 -channel model and dotted line to the 2-channel model fit.
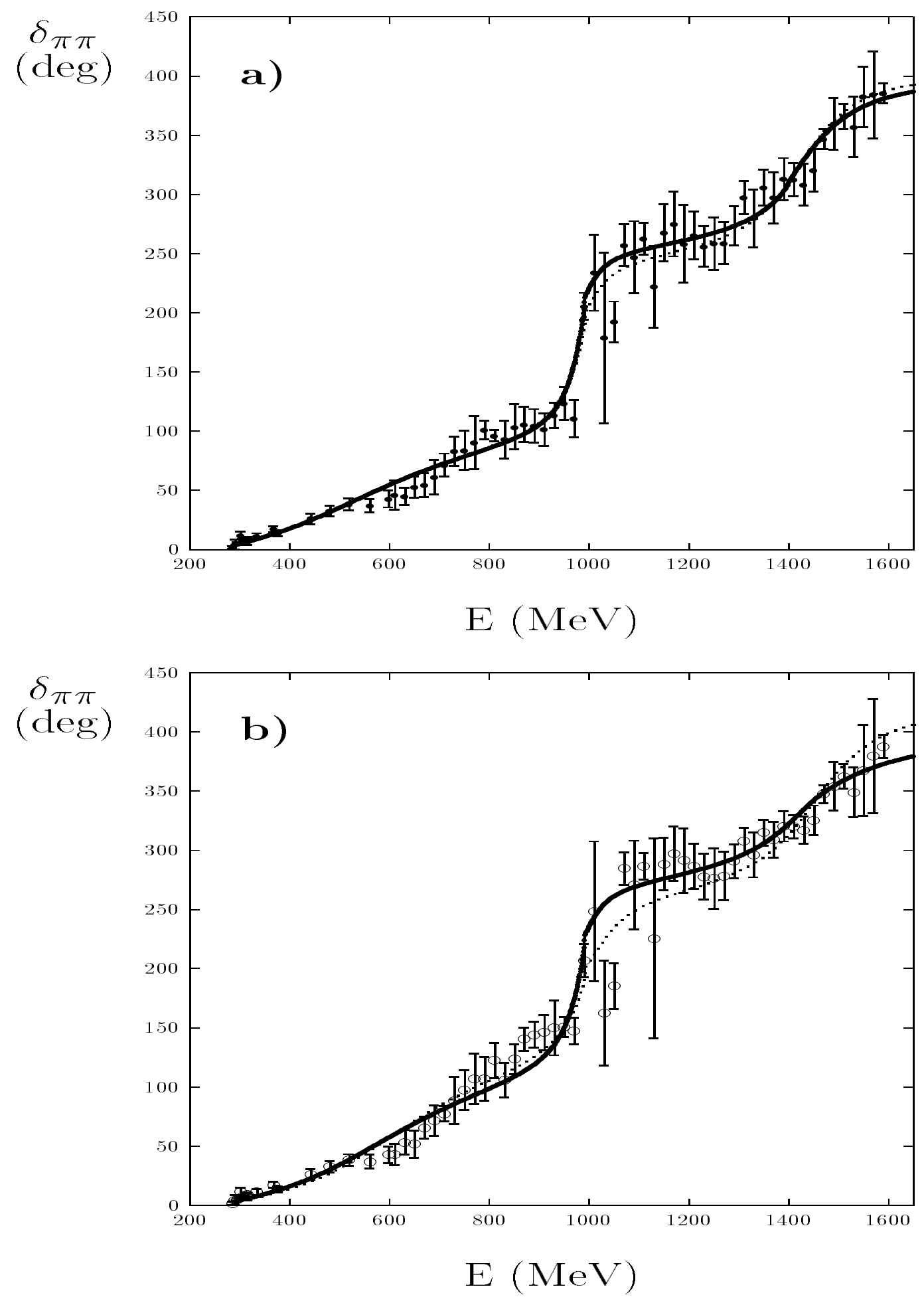
Figure 2: Energy dependence of inelasticity parameter $\eta_{\pi \pi}$ : a) fit to "downflat" data of [18], thick solid line corresponds to fit A, thin solid line to fit B and dotted line to the 2-channel model fit; b) fit to "up-flat" data of [18], thick solid line corresponds to fit $\mathrm{C}$, thin solid line to fit $\mathrm{D}$ and dotted line to the 2 -channel model fit.
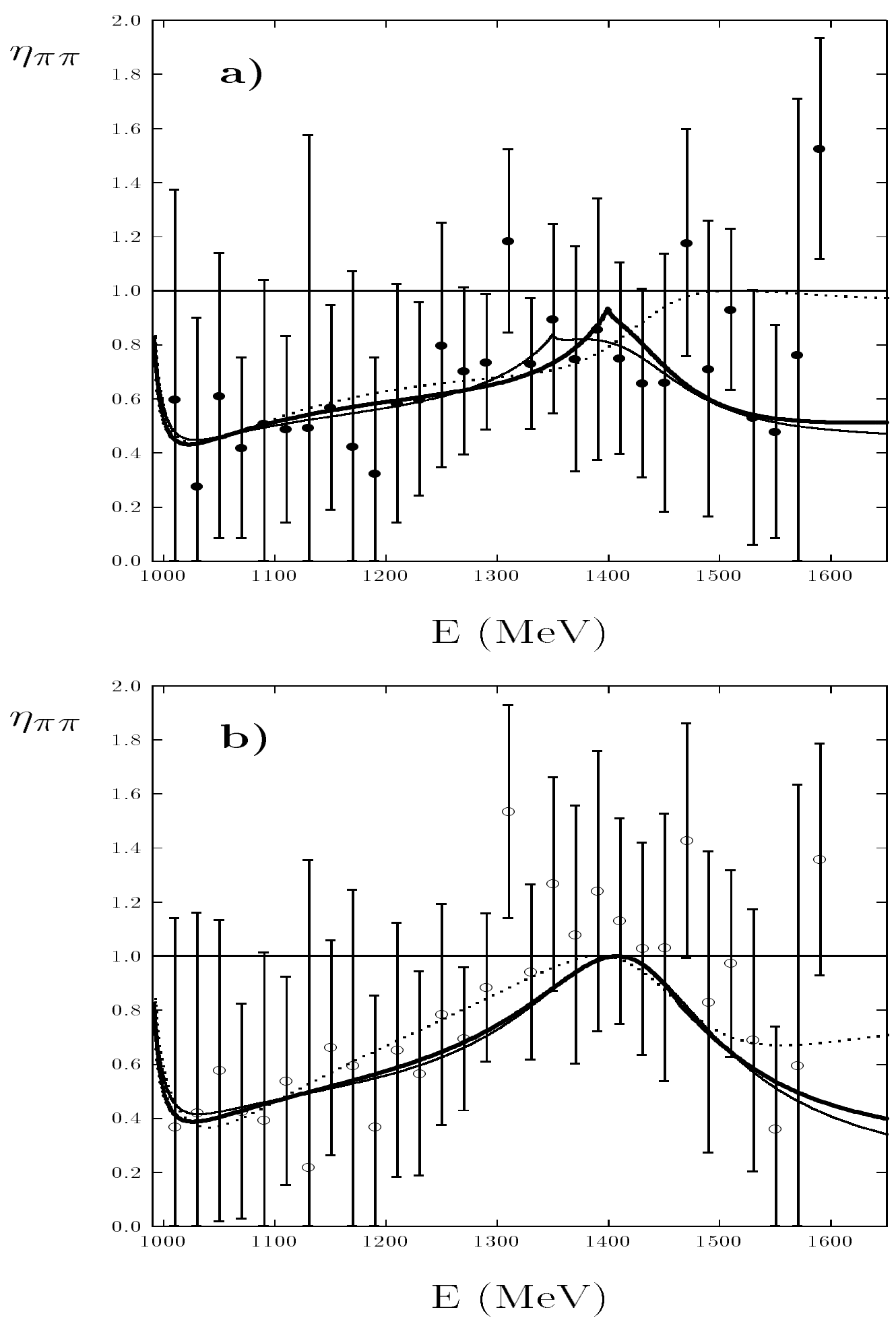
Figure 3: Energy dependence of phase shifts sum $\varphi_{\pi K}=\delta_{\pi \pi}+\delta_{K \bar{K}}$ : a) fit to "down-flat" data of [18], thick solid line corresponds to fit A, thin solid line to fit B and dotted line to the 2-channel model fit; b) fit to "up-flat" data, thick solid line corresponds to fit $\mathrm{C}$, thin solid line to fit $\mathrm{D}$ and dotted line to the 2 -channel model fit. Data for $\varphi_{\pi K}$ are taken from [17].
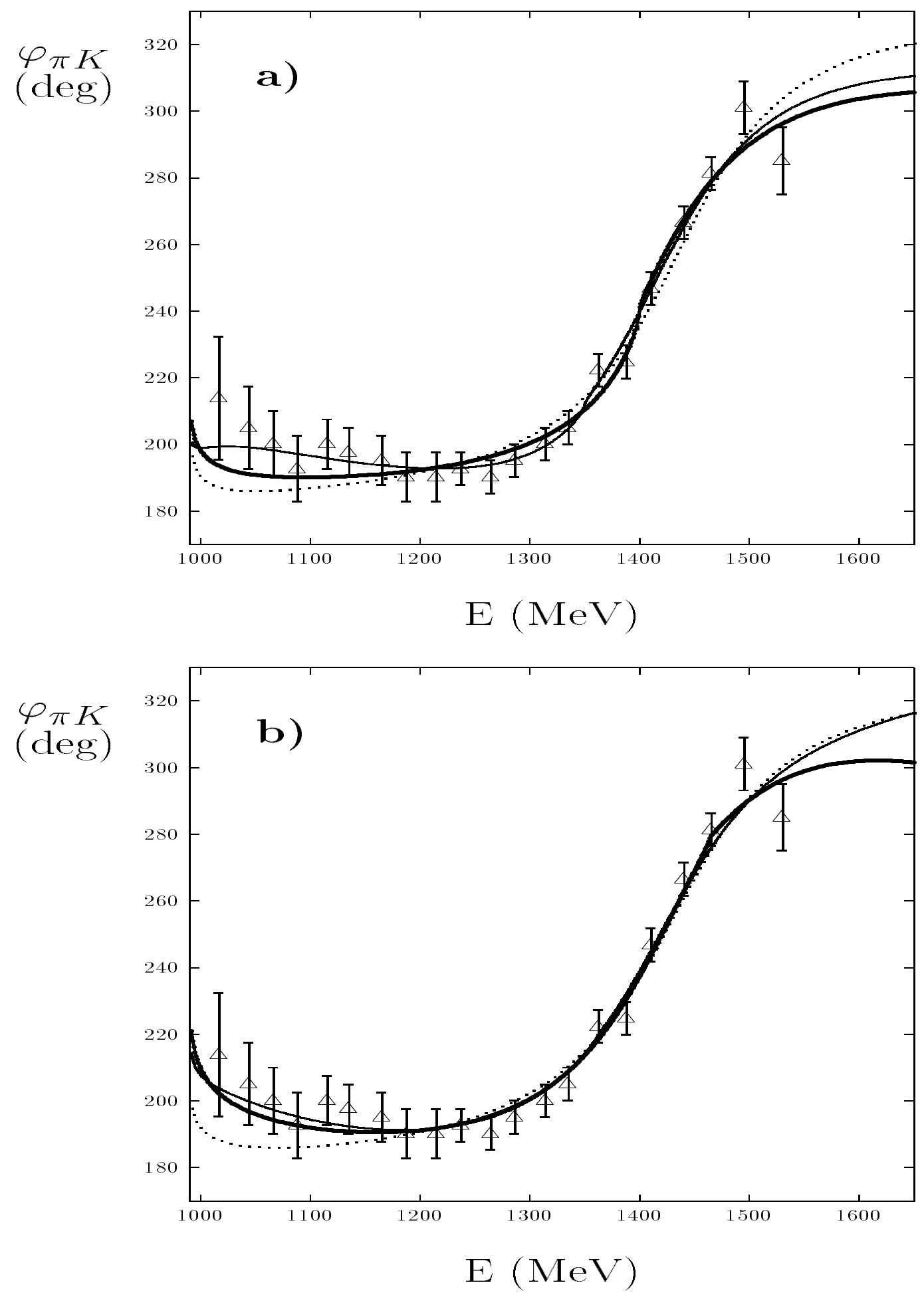
Table 1: Separable interaction parameters for 2- and 3-channel model fits to the "down-flat" and "up-flat" data from [18]. Values of $\beta$ and $m_{3}$ are given in GeV.

\begin{tabular}{|c|c|c|c|}
\hline Data & \multicolumn{3}{|c|}{ down-flat } \\
\hline model & 2-channel & \multicolumn{2}{|c|}{ 3-channel } \\
\hline fit & & $\mathrm{A}$ & $\bar{B}$ \\
\hline$\overline{\Lambda_{11,1}}$ & $-.14258 \times 10^{-3}$ & $-.29975 \times 10^{-2}$ & $-.52138 \times 10^{-2}$ \\
\hline$\Lambda_{11,2}$ & -.18895 & -.10844 & -.10552 \\
\hline$\Lambda_{22}$ & -.49106 & -.39304 & 3.1637 \\
\hline$\Lambda_{33}$ & 0 & $-.17447 \times 10^{-2}$ & $-.45719 \times 10^{-1}$ \\
\hline$\Lambda_{12,1}$ & $.27736 \times 10^{-5}$ & $.12039 \times 10^{-2}$ & $.10685 \times 10^{-1}$ \\
\hline$\Lambda_{12,2}$ & $.43637 \times 10^{-1}$ & -.11333 & -.80626 \\
\hline$\Lambda_{13,1}$ & 0 & $-.25841 \times 10^{-2}$ & $.14544 \times 10^{-4}$ \\
\hline$\Lambda_{13,2}$ & 0 & .39924 & $.21878 \times 10^{-2}$ \\
\hline$\Lambda_{23}$ & 0 & -.57955 & $-.17515 \times 10^{-1}$ \\
\hline$\beta_{1,1}$ & $3.0233 \times 10^{3}$ & $1.426 \times 10^{2}$ & $.81615 \times 10^{2}$ \\
\hline$\beta_{1,2}$ & 1.0922 & .92335 & .85776 \\
\hline$\beta_{2}$ & 2.2941 & 1.4959 & .47403 \\
\hline$\beta_{3}$ & & 1.3676 & $.45357 \times 10^{2}$ \\
\hline$m_{3}$ & & .70 & .67510 \\
\hline "Data & \multicolumn{3}{|c|}{ up-flat } \\
\hline model & 2-channel & \multicolumn{2}{|c|}{ 3-channel } \\
\hline fit & & $\mathrm{C}$ & $\mathrm{D}$ \\
\hline$\Lambda_{11,1}$ & $-.25550 \times 10^{-4}$ & $-.24649 \times 10^{-2}$ & $-.13063 \times 10^{-2}$ \\
\hline$\Lambda_{11,2}$ & -.15677 & $-.73339 \times 10^{-1}$ & $-.94483 \times 10^{-1}$ \\
\hline$\Lambda_{22}$ & -.66905 & .19475 & 3.6391 \\
\hline$\Lambda_{33}$ & 0 & $-.45073 \times 10^{-2}$ & -1.4588 \\
\hline$\Lambda_{12,1}$ & $-.23243 \times 10^{-5}$ & $.12710 \times 10^{-2}$ & $.10185 \times 10^{-2}$ \\
\hline$\Lambda_{12,2}$ & $.74350 \times 10^{-1}$ & -.32171 & -.85407 \\
\hline$\Lambda_{13,1}$ & 0 & $-.52567 \times 10^{-2}$ & $-.18043 \times 10^{-3}$ \\
\hline$\Lambda_{13,2}$ & 0 & 1.6134 & .17803 \\
\hline$\Lambda_{23}$ & 0 & -4.0462 & -.50484 \\
\hline$\beta_{1,1}$ & $1.6878 \times 10^{4}$ & $1.7379 \times 10^{2}$ & $3.2899 \times 10^{2}$ \\
\hline$\beta_{1,2}$ & 1.5882 & .92153 & .90097 \\
\hline$\beta_{2}$ & 1.4710 & .69032 & .30733 \\
\hline$\beta_{3}$ & & .23546 & 1.1419 \\
\hline$m_{3}$ & & .73252 & .68096 \\
\hline
\end{tabular}


Table 2: Best $\chi^{2}$ values of 2 - and 3-channel model fits for the "down-flat" and "up-flat" data of ref. [18]. Numbers of data points are indicated in parentheses. $\bar{\chi}^{2}$ values are obtained when fitting with reduced $\eta$ errors.

\begin{tabular}{|c|c|c|c|c|c|c|}
\hline Data & \multicolumn{3}{|c|}{ down-flat } & \multicolumn{3}{|c|}{ up-flat } \\
\hline Model & 2-channel & $3-\mathrm{ch}$ & nnel & 2-channel & $3-\mathrm{ch}$ & nnel \\
\hline fit & & $\overline{\mathrm{A}}$ & $\overline{\mathrm{B}}$ & & $\mathrm{C}$ & $\bar{D}$ \\
\hline$\chi_{\pi}^{2}(65)$ & 66.4 & 63.0 & 61.2 & 115.6 & 88.0 & 85.3 \\
\hline$\chi_{\pi+K}^{2}(21)$ & 26.3 & 15.9 & 9.7 & 27.0 & 13.1 & 13.2 \\
\hline$\chi_{n}^{2}(30)$ & 9.4 & 13.2 & 12.9 & 10.9 & 14.1 & 14.6 \\
\hline$\chi_{t o t}^{2}(116)$ & 102.1 & 92.1 & 83.8 & 153.5 & 115.2 & 113.1 \\
\hline${\overline{\chi_{\eta}}}^{2}(30)$ & 100.6 & 36.7 & 29.3 & 43.3 & 36.2 & 37.7 \\
\hline${\overline{\chi_{t o t}}}^{2}(116)$ & 193.2 & 115.6 & 100.1 & 186.0 & 137.3 & 136.2 \\
\hline
\end{tabular}

Table 3: Masses and widths of resonances found for the 2- and 3-channel fits to the "down-flat" and "up-flat" data

\begin{tabular}{|c|c|c|c|c|c|}
\hline \multicolumn{6}{|c|}{ 2-channel model } \\
\hline \multirow[t]{2}{*}{ pole } & \multirow[t]{2}{*}{ sheet } & \multicolumn{2}{|c|}{ down-flat } & \multicolumn{2}{|c|}{ up-flat } \\
\hline & & $M(\mathrm{MeV})$ & $\Gamma(\mathrm{MeV})$ & $M(\mathrm{MeV})$ & $\Gamma(\mathrm{MeV})$ \\
\hline$f_{0}(500)(\sigma)$ & -+ & 524.0 & 513.6 & 592.9 & 401.8 \\
\hline$f_{0}(980)$ & -+ & 993.4 & 79.3 & 1015.0 & 101.4 \\
\hline$f_{0}(1400)$ & -- & 1434.6 & 167.6 & 1429.7 & 179.2 \\
\hline \multicolumn{6}{|c|}{ "3-channel model } \\
\hline \multirow{3}{*}{ pole } & \multirow{3}{*}{ sheet } & \multicolumn{4}{|c|}{ down-flat } \\
\hline & & \multicolumn{2}{|c|}{$\mathrm{A}$} & \multicolumn{2}{|c|}{$\mathrm{B}$} \\
\hline & & $M(\mathrm{MeV})$ & $\overline{\Gamma(\mathrm{MeV})}$ & $M(\mathrm{MeV})$ & $\Gamma(\mathrm{MeV})$ \\
\hline$f_{0}(500)(\sigma)$ & -++ & 518.1 & 521.4 & 511.8 & 532.6 \\
\hline$f_{0}(980)$ & -++ & 989.0 & 62.0 & 992.4 & 68.2 \\
\hline$f_{0}(1400):$ & --- & 1405.1 & 147.8 & 1411.5 & 169.3 \\
\hline & --+ & 1456.4 & 93.3 & 1402.7 & 108.2 \\
\hline
\end{tabular}

\begin{tabular}{|c|c|c|c|c|c|}
\hline \hline \multirow{2}{*}{ pole } & \multirow{3}{*}{ sheet } & \multicolumn{4}{|c|}{ up-llat } \\
\cline { 3 - 6 } & & \multicolumn{2}{|c|}{$\mathrm{C}$} & \multicolumn{2}{|c|}{$\mathrm{D}$} \\
\cline { 3 - 6 } & $M(\mathrm{MeV})$ & $\Gamma(\mathrm{MeV})$ & $M(\mathrm{MeV})$ & $\Gamma(\mathrm{MeV})$ \\
\hline$f_{0}(500)(\sigma)$ & -++ & 561.7 & 466.8 & 558.2 & 477.6 \\
$f_{0}(980)$ & -++ & 992.2 & 67.6 & 995.3 & 71.7 \\
$f_{0}(1400):$ & --- & 1407.0 & 181.2 & 1418.8 & 179.3 \\
& --+ & 1423.8 & 177.4 & 1416.5 & 173.2 \\
\hline
\end{tabular}

